\title{
Cross- Sectional study of differences in phenomenology of acute psychosis with or without cannabis
}

\author{
Pal V. $\mathbf{S}^{1}$, Rastogi $\mathrm{P}^{2}$, Chauhan $\mathrm{A}^{3}$, Niranjan $\mathrm{V}^{4}$ \\ ${ }^{1}$ Dr. V. S. Pal, Associate Professor, ${ }^{2}$ Dr. Pali Rastogi, Assistant Professor, ${ }^{3}$ Dr. Amrita Chauhan, Post Graduate \\ Resident, ${ }^{4}$ Dr. Vijay Niranjan, Assistant Professor. All authors are affiliated with Department of Psychiatry \\ M.G.M Medical College Indore, MP, India.
}

Address Correspondence: Dr. Pali Rastogi, Assistant Professor, Department of Psychiatry, Mahatma Gandhi Memorial Medical College, A. B. Road, Indore, India. E-mail-dr.palirastogi73@gmail.com

\begin{abstract}
Background: Therehas been plethora of research regarding cannabis use disorder but very few studies reported demographic and phenomenological differences of acute psychosis with cannabis use to those of acute psychosis without cannabis use. This study attempted to evaluate the demographic and phenomenology differences between two groups of patients presenting with acute psychosis with cannabis use and acute psychosis without cannabis use. Material \& Method: Two group of patients recruited for study were 'Cases with Cannabis' and 'control without Cannabis' presenting with acute psychosis with preceding cannabis use and second one presenting with acute psychosis without preceding cannabis use in out-patient department of psychiatry, M.Y hospital, Indore Assessment done using rating scales. Results: Acute psychosis with cannabis wascharacterizedby primarily polymorphic clinical picture with predominance of positive and mood symptoms both in clear and disturbed sensorium. Acute psychosis without cannabis was characterized by mixed positive and negative symptoms in clear sensorium.In 'Cases with cannabis' group 96.7\% were males and 3.3\% were females,mean age was higher (33.7\%) than control (27.7\%) group. Conclusion: Acute psychosis with cannabis is characterized by primarily polymorphic clinical picture with predominance of positive and mood symptoms both in clear and disturbed sensorium. Acute psychosis without cannabis is characterized by mixed positive and negative symptoms. General symptoms of psychosis were also more in acute psychosis without cannabis.
\end{abstract}

Key words: Cannabis, Acute Psychosis, Cannabis Psychosis, Phenomenology

\section{Introduction}

The use of cannabis has been known to mankind for the time immemorial and has been deeply ingrained in Indian Culture exemplified by its heavy use on occasion "Mahashivaratri". The United Nations Office on Drugs and Crime (UNODC) reports that $3.9 \%$ of the global adult population uses cannabis. Data from the National Household Survey in India demonstrated a prevalence figure of $4 \%$ and $3.3 \%$ for lifetime and current cannabis users [1]. However, cannabis use has been widely reported to induce acute psychotic experiences, to affect the severity of psychotic symptoms, and previous meta-analyses have reported a 2-fold increase in the risk to develop a

Manuscript received: $4^{\text {th }}$ October 2017

Reviewed: $14^{\text {th }}$ October 2017

Author Corrected: $20^{\text {th }}$ October 2017

Accepted for Publication: 26 ${ }^{\text {th }}$ October 2017 psychotic disorder in cannabis users compared to nonusers [2]. The principal psychoactive compound in cannabis is delta-9-tetra hydrocannabinol, content of which varies in different preparation of cannabis [3]. Adverse mental health effects generally seen with more potent form of cannabis. THC and other cannabinoid agonist produce their effect by interacting with an endogenous cannabinnoid signaling system. There are two types of cannabinoid receptors. CB1 receptors found primarily in brainand mediate the psychological and behavioral effects of THC [4]. Cannabis is one of the environmental factor that has received attention in recent literature as possibly contributing to risk for psychotic disorder. Mostresearchers conclude to few type of psychosis associated with cannabis use: Acute toxic or 
organic psychosis, acute functional psychosis, chronic psychosis which persist after abstinence, amotivational syndrome and schizophrenia $S$ [5]. The study of cannabis effects on clinical course and outcome remains an interesting area both from academic and clinical point of view[6].

\section{Material \& Method}

Study Place-Study was conducted in department of psychiatry, MGM Medical College after clearance obtained from institutional ethic committee of MGMMC Indore.

Study design-The study was carried out with a Cross-sectionaldesign.

Sampling Method-30 patients were selected in each group with purposive sampling (Acute psychosis with cannabis use and acute psychosis without cannabis use) after satisfying inclusion criteria and taking informed consent.

\section{Inclusion criteria}

1. Patient or legally accepted relative giving written informed consent

2. Patients fulfilling criteria of acute psychosis with or without cannabis (As per ICD-10)

3. Patient aged 18-60 yrs

4. Patient taking alcohol not in dependence pattern

\section{Exclusion criteria}

Persons were excluded from participation in the study if they met any of the following:
1. Patient taking other substance excluding tobacco

2. Patient with mental retardation

3. Patient having organic brain disorder

4. Patient having life threatening medical condition

5. Patient having history of mental illness prior to onset of cannabis use in first group

We relied on self-reported data of cannabis use regarding pattern, type, duration, and amount of use which was further confirmed by keyrelatives. Data regarding cannabis use was collected using drug abuse screening test (DAST-20). Diagnosis in both groups made with ICD-10-DCR [7]. Patients were recruited from OPD, IPD and emergency department with purposive samplingandassessed with scales for comparison of phenomenologyusing PANSS (positive and negative syndrome scale), OAS (overt aggression scale) and YMRS (Young mania rating scale). Two group were assessed for socio-demographic variables with Semi structured data entry Performa.In two major groups these similar diagnosis matched for any differences crosssectionally.

Statistical Method- Data analysis done with spsssoftware. Data gathered was of non-normal distribution so we applied non-parametric test i.e., Mann-Whitney test to test the significance. Pvalue less than 0.05 was considered as statistically significant.

\section{Results}

In 'Cases with cannabis' group 96.7\% were malesand 3.3\% female was there in sample. While in 'Control without cannabis group' $63.3 \%$ males and 36\% females were there (Table-1). $36.7 \%$ of 'cases with cannabis' while $56.3 \%$ of 'controls without cannabis' belong to 21-30 years age group. These figures shows that psychosis is more common in 21-30 year of age group (Table-1). Mean age of 'cases with cannabis'came out to be 33.7 years while in 'control without cannabis' group it is 27.7 years. P value is 0.016 (Table-1). So there is significant difference between proportion of cases and control in different age group. 56.7\% of subjects from 'cases with cannabis' group reside in urban area and 40\% in rural area rest in semi urbanarea. While in 'control without cannabis' group 30\% subjects reside in both rural and urban area rest in semi urban area. In both groups more than $50 \%$ of subjects belongs to nuclear family type. In 'Cases with cannabis' group 70\% of patient were educated up to $10^{\text {th }}$ standard while 'Control withoutcannabis' group 50\% of patient educated up to $10^{\text {th }}$ standard and $13.3 \%$ were illiterate. In 'Cases with cannabis' group $30 \%$ of patient engaged in skilled and semiskilled occupation while $26.7 \%$ in same occupation in 'Control without cannabis' group. Baseline mean positive score (at 0 day) in 'case with cannabis' group was 29.7 and of 'control without cannabis' group was 23 (Table-2).

Significant difference in baseline mean positive scores between two groups with p value of 0.000 suggestive of prominence of positive symptom characterized by high score on excitement, grandiosity, suspiciousness and hostility on PANSS in 'cases with cannabis' groupas compared to 'control without cannabis' group. Baseline mean negative score in 'case with cannabis' group is 16.2, in 'control without cannabis' group is 20.6 (Table-3). 
So there was significant difference in mean negative score between two groups with $\mathrm{P}$ value of 0.011 indicative of prominence of negative symptomin 'control without cannabis' group as compared to 'cases with cannabis'group.

Table-1: Sex \&age distribution $\&$ mean age distribution of case and control.

\begin{tabular}{|c|c|c|c|c|}
\hline \multirow[t]{2}{*}{ Gender } & \multicolumn{2}{|c|}{$\operatorname{Cases}(\mathrm{N}=30)$} & \multicolumn{2}{|c|}{ Control(N=30) } \\
\hline & $\mathbf{N}$ & $\%$ & $\mathbf{N}$ & $\%$ \\
\hline Male & 29 & 96.7 & 19 & 63.3 \\
\hline Female & 1 & 3 & 11 & 36 \\
\hline \multicolumn{5}{|l|}{ Age } \\
\hline $16-20$ & 3 & 10.0 & 4 & 13.3 \\
\hline $21-30$ & 11 & 36.7 & 17 & 56.7 \\
\hline $31-40$ & 8 & 26.7 & 7 & 23.3 \\
\hline $41-50$ & 5 & 16.7 & 2 & 6.7 \\
\hline $51-60$ & 3 & 10.0 & 0 & 0.0 \\
\hline Groups & Mean & Min & $\operatorname{Max}$ & 'p' value \\
\hline Cases & 33.7 & 19.0 & 55.0 & \multirow{2}{*}{0.016} \\
\hline Control & 27.7 & 15.0 & 45.0 & \\
\hline
\end{tabular}

Table- 2: Comparative PANSS Positive subscale scores between cases andcontrol.

\begin{tabular}{|c|c|c|c|c|c|}
\hline Groups & Mean & Min & Max & 土SD & P value \\
\hline Cases & 29.7 & 14.0 & 47.0 & 7.4 & 0.000 \\
\hline Control & 23.0 & 13.0 & 37.0 & 6.0 & \\
\hline
\end{tabular}

Table- 3:Comparative PANSS Negative subscale scores between cases and control.

\begin{tabular}{|c|c|c|c|c|c|}
\hline Groups & Mean & Min & Max & $\mathbf{\pm S D}$ & 'p' value \\
\hline Cases & 16.2 & 8.0 & 36.0 & 7.9 & 0.011 \\
\hline Control & 20.6 & 7.0 & 36.0 & 7.6 & \\
\hline
\end{tabular}

Table- 4:Comparative PANSS General subscale score between cases and control.

\begin{tabular}{|c|c|c|c|c|c|}
\hline Groups & Mean & Min & Max & \pm SD & 'p'value \\
\hline Cases & 35.4 & 20.0 & 59.0 & 9.2 & 0.005 \\
\hline Control & 43.4 & 23.0 & 61.0 & 8.5 & \\
\hline
\end{tabular}

Table- 5:Comparative Overt Aggression scale scores between cases and control.

\begin{tabular}{|c|c|c|c|c|c|}
\hline Groups & Mean & Min & Max & 土SD & 'p'value \\
\hline Cases & 19.3 & 0.0 & 67.0 & 15.8 & 0.001 \\
\hline Control & 9.6 & 0.0 & 44.0 & 9.4 & \\
\hline
\end{tabular}

Table- 6: Comparative YMRS scale mean scores between cases and control.

\begin{tabular}{|c|c|c|c|c|c|}
\hline Groups & Mean & Min & Max & 土SD & 'p'value \\
\hline Cases & 30.9 & 0.0 & 52.0 & 12.3 & $0.000 \mathrm{HS}$ \\
\hline Control & 13.0 & 6.0 & 36.0 & 8.3 & \\
\hline
\end{tabular}

Baseline mean general score in 'case with cannabis' group was 35.4 and in 'control without cannabis' group was 43.4 (Table-4). Difference of score between two groups was significant. This indicate that general symptom of psychosis were more in 'control without cannabis' group as compared to 'cases with cannabis' group. Predominant symptom in general psychopathology scale were anxiety, guiltfeelings, depression, tension, active 
social avoidance in 'control without cannabis' group while disorientation, poor, judgement, attention, unusual thought content, uncooperativeness, poor impulse control were common among 'cases with cannabis'group. Baseline mean Aggression score in 'case with cannabis' group was 19.3 which is more than mean 9.6 of'control without cannabis' group. $\mathrm{P}$ value is 0.01 , so this difference between two group wassignificant. This indicate aggression was more in 'cases with cannabis' using group as compared to 'control without cannabis' group (Table-5). Baseline Mean YMRS score in 'case with cannabis' group was 30.9 which is more than mean 13 of control group. $\mathrm{P}$ value is 0.000 , so this difference between two groups is highly significant. This indicate mood symptoms were more common in 'cases with cannabis' group as compared to control group (Table-6).

\section{Discussion}

Among 'cases with cannabis' group, 29 cases $(96.7 \%)$ were male and one case $(3.3 \%)$, was female. This could be attributed to low prevalence of substance abuse among females in general population in India and cannabis in particular. This finding was consistent with previous studies like Varma et al concluded in his study that prevalence for cannabis abuse in female is very less [8].

$36.7 \%$ of 'cases with cannabis' while $56.7 \%$ of 'controls without cannabis' belong to 21-30 years age group. These figures shows that psychosis is more prevalent among 21-30 year of age group population. Mean age of psychosis in 'cases with cannabis' group was 33.7 years while in 'control without cannabis' group it was 27.7 years. There was significant difference between proportion of cases and control in different age group and which is also consistent with finding of previous studies. Varma concludedin his study of cannabis psychosis that mean age of patients of cannabis psychosis is 35.81 years which is higher than average mean of 32 years for other mental patient [8].

Baseline mean positive scorein 'cases with cannabis' group was 29.7 and of 'control without cannabis' group was 23. Significant difference in baseline mean positive scores between two groups is suggestive of prominence of positive symptom in 'cases with cannabis' group as compared to 'control without cannabis' group. Higher mean positive score in 'cases with cannabis' group was consistent with results from study by R Patel et al [9]. Baseline mean negative score in 'case with cannabis' group was 16.2, in 'control without cannabis' group was 20.6. There was significant difference in mean negative score between two groups indicative of prominence of negative symptom in 'control without cannabis' group. Carol. $\mathrm{M}$ et al study results also onbserved higher negative symptom score (14.16vs 11.67) of PANSS sub scale on comparing primary psychosis with substance induced psychosis [9]. Baseline mean general score in 'case with cannabis' group was 35.4 and in 'control without cannabis' group was 43.4. Difference of score between two groups was significant. This indicate that general symptom of psychosis were more in 'control without cannabis' group as compared to 'cases with cannabis' group. Similar results were reported by Carol.M et al [10].

Baseline mean Aggression score in "case with cannabis' group was 19.3 which is more than mean 9.6 of 'control without cannabis' group. This difference between two groups was significant. This indicate aggression was more in 'cases with cannabis' using group as compared to 'control without cannabis' group. This finding was consistent with other studies [9].

Baseline Mean YMRS score in 'case with cannabis' group was 30.9 which is more than mean 13 of control group. This difference between two groupswas highly significant. This indicate mood symptoms were more common in'cases with cannabis' group as compared to control group. Similar results were reported by Debasish Basu et al from India [11].

\section{Conclusion}

This prospective case control study concludes that Acute psychosis with cannabis is characterized by primarily polymorphic clinical picture with predominance of positive and mood symptoms both in clear and disturbed sensorium. Acute psychosis without cannabis is characterized by mixed positive and negative symptoms. General symptoms of psychosis were also more in acute psychosis without cannabis. The important limitations of the study were of small sample size, cross sectional assesement, purposive sampling and institutional setting. Further research is warranted regarding phenomenology, course and outcome of psychosis associated with Cannabis use. 
What study adds to Existing knowledge- Very few studies reported from India that differentiate the clinical feature of cannabis psychosis from other acute psychosis which may have an important implication in diagnosis and treatment part of illness. Our study gave a clear insight in to the phenomenology of psychosis associated with cannabis use and that would be helpful in elucidating role of Cannabis in modifying psychosis, which has been a challenging task for specialist in field.

\section{Recommendations}

- There is dearth of research regarding phenomenology of psychosis associated with cannabis use, particularly from India, warranting further research to find out role of cannabis in psychosis.

- Government should implement strict rules towards illicit drug trafficking, particularly of Cannabis.

- Awareness should be raised at community level by social workers using mass media programmes regarding adverse mental health effects of cannabis use.

Author contribution- This study conducted under guidance of DrV.S Pal (Associate Professor) with valuable contribution from Dr. Pali Rastogi (Assistant Professor) in timely evaluation of study.Patient interviewing and data collection done by Dr Amrita chauhan (PG Resident) in collaboration with Dr Vijay Niranjan (Assistant Professor) doing data analysis and manuscript preparation.

\section{Funding: Nil, Conflict of interest: None Permission of IRB: Yes}

\section{References}

1. World Health Organization. Cannabis: a health perspective and research agenda. (1997).

2. Marconi A, Di Forti M, Lewis CM, Murray RM, VassosE.Meta-analysis of the Association Between the Level of Cannabis Use and Risk ofPsychosis. Schizophr Bull. 2016 Sep;42(5):1262-9. doi: 10.1093/schbul/ sbw 003. Epub 2016 Feb 15.

3. Mackie K.Distribution of cannabinoid receptors in the central and peripheral nervous system. HandbExp Pharmacol. 2005;(168):299-325.

4. De Petrocellis L, Cascio MG, Di Marzo V. The endocannabinoidsystem: a generalview and latest additions. Br J Pharmacol. 2004 Mar;141(5):76574. Epub 2004 Jan 26.

5. Johns A. Psychiatric effects of cannabis. Br J Psychiatry. 2001 Feb;178:116-22.

6. Haobam M, Mohanty R, Senjam G, Heramani N. Cannabis and its associated psychopathology. Journal of Medical Society. 2017 Sep 1;31(3):143.

7. Cooper JE, World Health Organization. Pocket Guide to the ICD-10 Classification of Mental and Behavioural Disorders: With Glossary and Diagnostic Criteria for Research: ICD-10/DCR-10. American Psychiatric Pub; 1994.

8. Varma LP. Cannabis psychosis. Indian Journal of Psychiatry. 1972 Jul 1;14(3):241.

9. Patel R, Dominguez MD, Fisher HL, Johnson S, Hodes M. EPA-1645-The impact of cannabis use on clinical presentation in first-episode psychosis: adolescent-versus adult-onset psychosis. European Psychiatry. 2014 Dec 31;29:1.

10. Caton CL, Drake RE, Hasin DS, Dominguez B, Shrout PE, Samet S, SchanzerB.Differences between early-phaseprimarypsychotic disorders with concurrentsubstance use and substanceinduced psychoses. Arch Gen Psychiatry. 2005 Feb;62(2):137-45.

11. Basu D, Malhotra A, Bhagat A, Varma VK.Cannabispsychosis and acuteschizophrenia. a case-control study from India. Eur Addict Res. 1999 Jun;5(2):71-3.

\section{How to cite this article?}

Pal V. S, Rastogi P, Chauhan A, Niranjan V. Cross-Sectional study of differences in phenomenology of acute psychosis with or without cannabis. Int J Med Res Rev 2017;5(10):883-887.doi:10.17511/ijmrr. 2017.i10.02 